\title{
Disseminated adenovirus infection in a 10-year-old renal allograft recipient
}

\author{
Bora Lee ${ }^{1}$, Eujin Park ${ }^{2}$, Jongwon $\mathrm{Ha}^{3}$, II Soo Ha ${ }^{4}$, Hae II Cheong ${ }^{4}$, Hee Gyung Kang ${ }^{4}$ \\ ${ }^{1}$ Department of Pediatrics, Seoul National University College of Medicine, Seoul, Korea \\ ${ }^{2}$ Department of Pediatrics, Hallym University Medical Center, Seoul, Korea \\ ${ }^{3}$ Department of Surgery, Seoul National University College of Medicine, Seoul, Korea \\ ${ }^{4}$ Division of Nephrology, Department of Pediatrics, Seoul National University College of Medicine, Seoul, Korea
}

\begin{abstract}
Disseminated adenovirus infection can result in high mortality and morbidity in immunocompromised patients. Here, we report the case of a 10 -year-old renal allograft recipient who presented with hematuria and dysuria. Adenovirus was isolated from his urine. His urinary symptoms decreased after intravenous hydration and reduction of immunosuppressants. However, 2 weeks later he presented with general weakness and laboratory tests indicated renal failure necessitating emergency hemodialysis. Adenovirus was detected in his sputum; therefore, intravenous ganciclovir and immunoglobulin therapy were initiated. Renal biopsy revealed diffuse necrotizing granulomatous tubulointerstitial nephritis compatible with renal involvement of the viral infection. Adenovirus was detected in his serum. Despite cidofovir administration for 2 weeks, adenovirus was also detected in the cerebrospinal fluid, resulting in generalized tonic-clonic seizure. The patient died 7 weeks after the onset of urinary symptoms. Adenovirus should be considered in screening tests for post-renal transplantation patients who present with hemorrhagic cystitis.
\end{abstract}

Keywords: Adenoviridae, Kidney transplantation, Opportunistic infections, Pediatrics

\section{Introduction}

Adenoviruses are one of the most common pathogens that cause febrile illness in the young population. They commonly infect the epithelium of the respiratory system but can also cause genitourinary, gastrointestinal, ophthalmologic, neurologic, and disseminated diseases. Most adenoviral infections are self-limiting and are re-

\footnotetext{
Received May 28, 2018; Revised August 6, 2018;

Accepted August 16, 2018

Correspondence: Hee Gyung Kang

Division of Nephrology, Department of Pediatrics, Seoul National University

College of Medicine, 101 Daehak-ro, Jongno-gu, Seoul 03080, Korea.

E-mail: kanghg@snu.ac.kr

ORCID: https://orcid.org/oooo-0001-8323-5320

Copyright (C) 2018 by The Korean Society of Nephrology

(c) This is an open-access article distributed under the terms of the Creative Commons Attribution Non-Commercial License (http://creativecommons.org/licenses/bync-nd/4.0/), which permits unrestricted non-commercial use, distribution, and reproduction in any medium, provided the original work is properly cited.
}

solved within 2 weeks; however, immunocompromised hosts are more susceptible to adenoviral infections and show a significantly higher mortality rate than healthy patients [1].

Although potent immunosuppressive agents have helped reduce the incidence of transplant organ rejection, they increase the patient's susceptibility to opportunistic infections as well as cancer [2]. Opportunistic infections are more likely to occur between the first and sixth month after organ transplantation [3]. Adenovirus infection is a well-known cause of mortality after bone marrow transplantation [4]. However, limited data are available regarding adenovirus infections following solid organ transplantations, such as renal allograft transplantation. This report describes the case of a pediatric patient with fatal disseminated adenovirus infection after renal transplantation. 
Lee, et al. Adenovirus infection after renal transplantation

\section{Case report}

The patient was a 10-year-old boy who was born preterm at a gestational age of 33 weeks and 2 days. He exhibited developmental delays in growth and motor function. He first visited our center for evaluation of short stature at the age of 6 years. He was clinically diagnosed with Russell-Silver syndrome, and proteinuria was incidentally found along with hypoalbuminemia. Conventional treatment for nephrotic syndrome, including high-dose steroid and calcineurin inhibitor, had been ineffective and his renal function had deteriorated. At the age of 8 years, he suffered from right renal vein thrombosis and pulmonary embolism for which he had been treated with anticoagulants. Despite treatment, he developed persistent pulmonary hypertension, which was managed with sildenafil. He underwent living donor kidney transplantation at the age of 9 years, with his mother as the donor. His renal function was well maintained with immunosuppression using prednisolone, mycophenolate mofetil (MMF), and tacrolimus. He discontinued MMF about 70 days after the transplantation and steroid was tapered. Tacrolimus was continued with blood levels maintained at approximately $10 \mathrm{ng} / \mathrm{mL}$. About 3 months after the transplantation, he developed pneumocystis pneumonia that necessitated mechanical ventilation and was treated with intravenous sulfamethoxazole/trimethoprim for 14 days. Steroid was increased (from $2.5 \mathrm{mg}$ to $20 \mathrm{mg}$ prednisolone) and continued at the same dose after discharge. On the 135th postoperative day, he presented with dysuria and gross hematuria. Biochemistry showed the following: blood urea nitrogen (BUN), $24 \mathrm{mg} /$ $\mathrm{dL}$; creatinine $(\mathrm{Cr}), 0.56 \mathrm{mg} / \mathrm{dL}$; and C-reactive protein (CRP), $0.42 \mathrm{mg} / \mathrm{dL}$. Urinalysis revealed a red blood cell $(\mathrm{RBC})$ count $>100 /$ high power fields (HPF) and a white blood cell (WBC) count > 100/HPF. Urine culture for bacteria and BK virus were negative; however, urine John Cunningham (JC) virus polymerase chain reaction (PCR) and urine adenovirus culture were positive (1-9 cells/ HPF). He was diagnosed with hemorrhagic cystitis and was managed with hydration and pain control because of tolerable dysuria and absence of fever. After a week, dysuria and hematuria persisted with a RBC count $>100 /$ HPF and WBC count 1 to 4/HPF; however, his immunosuppression was maintained.

Three weeks later, he visited the emergency room with
Table 1. Clinical characteristics of each admission

\begin{tabular}{lcc}
\hline Time after transplantation & 135th day & 159th day \\
\hline Tacrolimus level $(\mathrm{ng} / \mathrm{mL})$ & 3.7 & 16.3 \\
BUN $(\mathrm{mg} / \mathrm{dL})$ & 24 & 175 \\
Creatinine $(\mathrm{mg} / \mathrm{dL})$ & 0.56 & 8.29 \\
AST/ALT $(\mathrm{IU} / \mathrm{L})$ & $31 / 50$ & $30 / 24$ \\
Bilirubin, total $(\mathrm{mg} / \mathrm{dL})$ & 0.4 & 0.2 \\
CRP $(\mathrm{mg} / \mathrm{dL})$ & 0.42 & 30.23 \\
Total $\mathrm{CO}_{2}(\mathrm{mmol} / \mathrm{L})$ & 16 & 15 \\
PT $(\mathrm{INR})$ & - & 0.92 \\
aPTT $(\mathrm{sec})$ & - & 33.1 \\
\hline
\end{tabular}

ALT, alanine aminotransferase; aPTT, activated partial thromboplastin time; AST, aspartate aminotransferase; BUN, blood urea nitrogen; CRP, C-reactive protein; PT, prothrombin time; aPT, activated partial thromboplastin time.

fever, general weakness, chest tightness, mild cough with persistent dysuria, and hematuria. Laboratory tests revealed BUN at $175 \mathrm{mg} / \mathrm{dL}, \mathrm{Cr}$ at $8.29 \mathrm{mg} / \mathrm{dL}$, and CRP at $30.23 \mathrm{mg} / \mathrm{dL}$ (Table 1). He underwent emergent hemodialysis and was empirically treated with piperacillin/tazobactam considering the possibility of urosepsis. Sputum, blood, and urine culture results were negative; however, the adenovirus real-time PCR of sputum and blood cytomegalovirus (CMV) antigen were positive. He was presumed to have disseminated adenovirus infection and was treated with immunosuppression reduction and ganciclovir. Renal allograft biopsy showed diffuse necrotizing granulomatous tubulointerstitial nephritis (Fig. 1), indicating infectious tubulointerstitial nephritis rather than rejection. Staining for CD3 and C4d was negative, indicating negativity for cellular and humoral rejection, respectively. He also tested positive for JC virus PCR and serum CMV PCR, suggesting coinfection. He was treated with ganciclovir targeting CMV, antibiotic therapy with renal impairment dose for CMV infection, granulocyte colony-stimulating factor, immunoglobulin, transfusion, and hemodialysis; however, the inflammatory marker and fever remained consistently high.

Cidofovir is known to be more effective than other antiviral agents against adenovirus infection [5]. Therefore, despite its well-known nephrotoxicity, we initiated cidofovir treatment on the 167 th postoperative day because of persistent infection. The patient was given cidofovir ( 1 $\mathrm{mg} / \mathrm{kg} /$ day, 3 times/week without probenecid), a renal impairment dose of post-hematopoietic stem cell transplant adenovirus infection induction treatment, with on- 

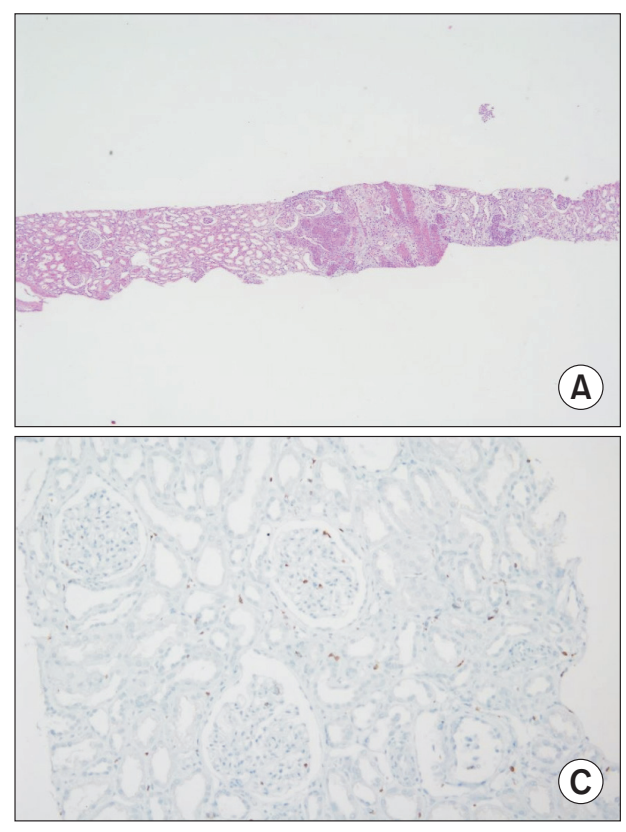
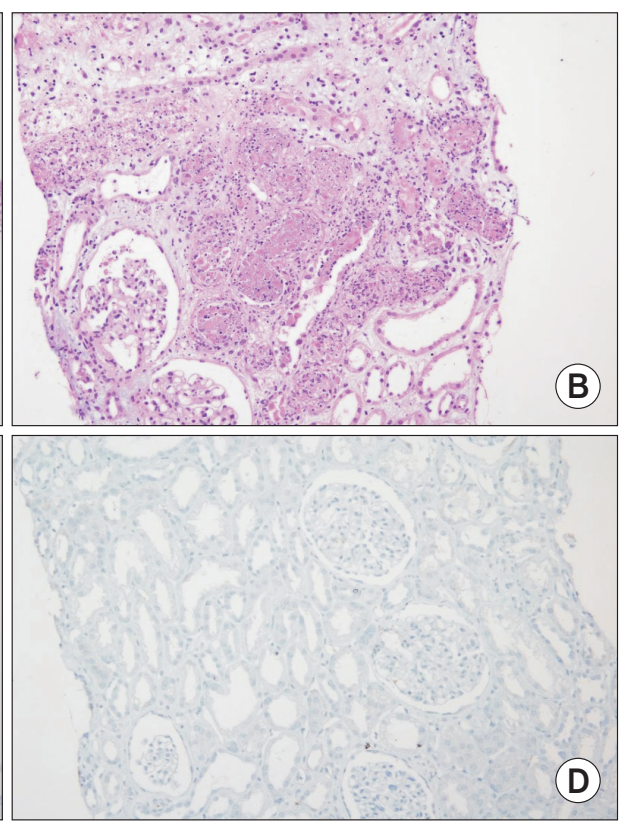

Figure 1. Renal allograft biopsy. (A) Alternating necrotizing granulomatous tubulointerstitial nephritis $(\times 20)$. (B) Necrosis of glomerular and tubular system (H\&E stain, $\times 200)$. (C) No CD3 deposition which means negative for cellular rejection (immunoperoxidase stain, $\times 200$ ). (D) No C4d deposition which means negative for humoral rejection (immunoperoxidase stain, $\times 200$ ).

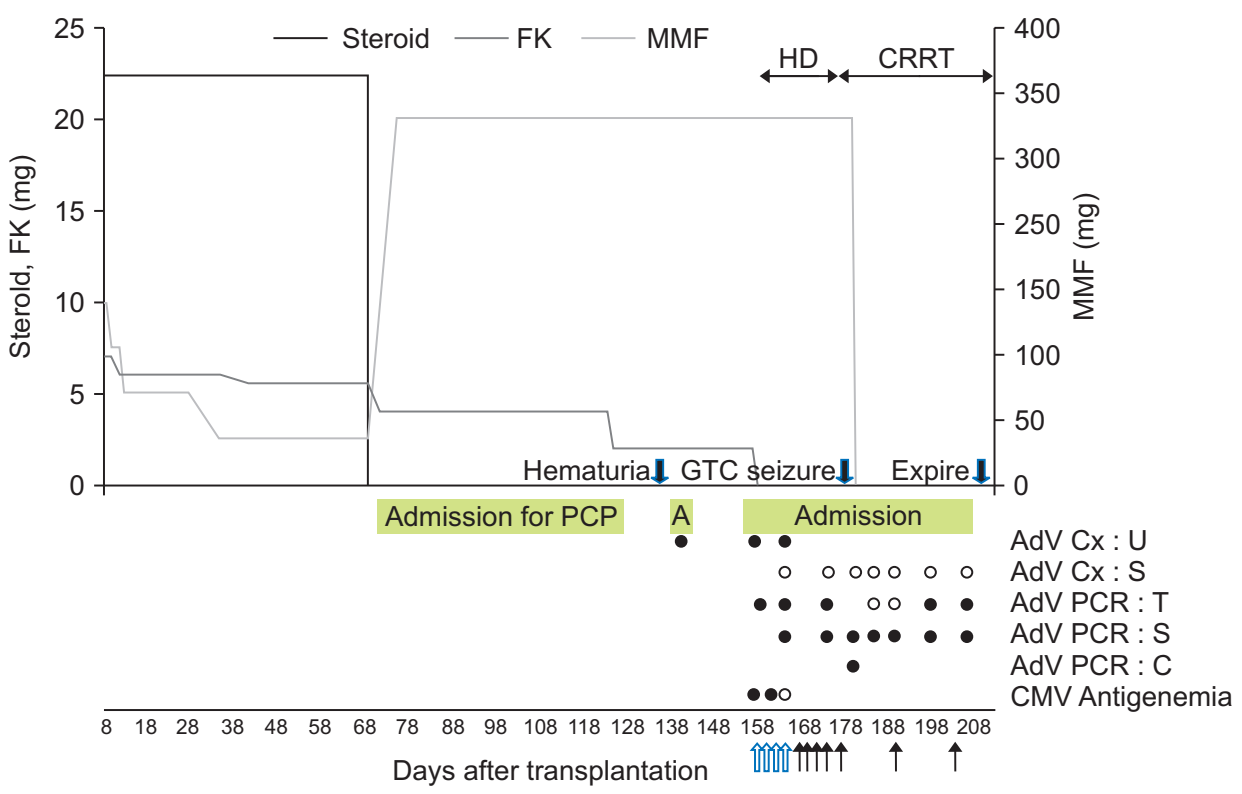

Figure 2. Time line and clinical course after transplantation. Positive (closed symbols) and negative (open symbols) results of culture (Cx) and polymerase chain reaction (PCR) of urine (U), serum $(\mathrm{S})$, throat swab $(\mathrm{T})$, and cerebrospinal fluid (C) specimens for detection of adenovirus (AdV). The white and black arrows indicate intravenous immunoglobulin and cidofovir, respectively.

A, admission; CMV, cytomegalovirus; CRRT, continuous renal replacement therapy; FK, tacrolimus; GTC seizure, generalized tonic-clonic seizure; HD, hemodialysis; MMF, mycophenolate mofetil; $\mathrm{PCP}$, pneumocystis pneumonia.

going hemodialysis. After administration of 4 doses, we changed the regimen to $2 \mathrm{mg} / \mathrm{kg}$ every 2 weeks as a renal impairment dose for maintenance therapy, of which 3 treatments were completed. However, serum adenovirus PCR and cerebrospinal fluid adenovirus PCR were positive, his renal function was not recovered, and he presented generalized tonic-clonic seizure. Although treatment with vancomycin, meropenem, and acyclovir was initiated to control meningitis, his general condition continued to deteriorate. Anemia, leukopenia, and thrombocytopenia developed; these could be attributed to bone marrow suppression associated with severe infection. Despite intensive care, including mechanical ventilation and continuous renal replacement therapy, he died on the 215th day post-renal transplantation. Fig. 2 shows the summary of his clinical course and treatment.

\section{Discussion}

Adenovirus infection is defined as detection of adenovirus by immunofluorescence, culture, histology or PCR from any site. Asymptomatic adenovirus infection refers 
to the absence of clinical symptoms whereas adenovirus disease is defined as anatomically corresponding symptoms in the absence of any other identifiable cause. Disseminated adenovirus disease is an adenovirus infection affecting two or more organs or systems [6]. Adenovirus infections after solid-organ transplantation may be asymptomatic or manifested as disseminated and severe infection with prolonged viral propagation and significant mortality and morbidity, including graft dysfunction and rejection [7]. This was the first case of disseminated adenovirus infection that led to mortality among all cases of pediatric renal transplantation treated at our center. Infection may be associated with the reactivation of a latent infection in the graft or the recipient or with a novel infection. Pre-transplant infection screening does not include adenovirus screening; therefore, we do not have information regarding the presence of a potential preexisting adenovirus infection in our patient.

Establishing a diagnosis of adenovirus infection can be challenging; infection is diagnosed when a culture or PCR is positive but it may be 3 weeks before the cytopathic changes become detectable. Quantitative real-time PCR is useful during follow-up because the presence of $>1 \times 10^{6}$ copies is associated with increased mortality [8].

Adenovirus infection in solid organ transplantation recipients usually involves the donor organ. In renal transplantation recipients, the primary clinical presentation is acute hemorrhagic cystitis, sometimes complicated with interstitial nephritis [9]. To our knowledge, no controlled trials highlighting the benefit of any antiviral agent in human adenoviral disease have been conducted. So far, cidofovir appears to be more effective against adenovirus in vitro than any other antiviral agent; however, nephrotoxicity is a major adverse effect of cidofovir. In this patient, cidofovir was given at least a week after the infection became disseminated; however, earlier administration of cidofovir might have been more effective. In addition, it would have been useful to measure the serum viral load of adenovirus to assess the disease activity and the effect of medication.

Adenoviral infections in organ transplantation patients can result in fatal complications and mortality. Therefore, adenovirus should be considered and included in all laboratory screening tests for post-renal transplantation patients with hemorrhagic cystitis. Prospective studies are warranted to better understand the pathogenesis and to develop appropriate screening and therapeutic guidelines for adenovirus infections in transplant recipients.

\section{Conflicts of interest}

All authors have no conflicts of interest to declare.

\section{References}

[1] Hierholzer JC. Adenoviruses in the immunocompromised host. Clinl Microbiol Rev 5:262-274, 1992

[2] Halloran PF. Immunosuppressive drugs for kidney transplantation. N Eng J Med 351:2715-2729, 2004

[3] Fishman JA, Rubin RH. Infection in organ-transplant recipients. N Engl J Med 338:1741-1751, 1998

[4] Howard DS, Phillips II GL, Reece DE, et al. Adenovirus infections in hematopoietic stem cell transplant recipients. Clin Infect Dis 29:1494-1501, 1999

[5] Neofytos D, Ojha A, Mookerjee B, et al. Treatment of adenovirus disease in stem cell transplant recipients with cidofovir. Biol Blood Marrow Transplant 13:74-81, 2007

[6] Tebruegge M, Curtis N. Adenovirus infection in the immunocompromised host. Adv Exp Med Biol 659:153-174, 2010

[7] Florescu D, Hoffman J. Adenovirus in solid organ transplantation. Am J Transplant 13 (Suppl 4):206-211, 2013

[8] Claas EC, Schilham MW, de Brouwer CS, et al. Internally controlled real-time PCR monitoring of adenovirus DNA load in serum or plasma of transplant recipients. J Clin Microbiol 43:1738-1744, 2005

[9] Ito M, Hirabayashi N, Uno Y, Nakayama A, Asai J. Necrotizing tubulointerstitial nephritis associated with adenovirus infection. Hum Pathol 22:1225-1231, 1991 